\title{
Epidemiology of Cervical Spine Fractures
}

\author{
Mahnaz Yadollahi, ${ }^{1}$ Shahram Paydar, ${ }^{1,3}$ Haleh Ghaem, ${ }^{4}$ Mohammad Ghorbani, ${ }^{2,}$ ' Seyed Mohsen \\ Mousavi, ${ }^{3}$ Ali Taheri Akerdi, ${ }^{3}$ Eimen Jalili, ${ }^{1}$ Mohammad Hadi Niakan, ${ }^{1,3}$ Hossein Ali Khalili, ${ }^{1}$ Ali \\ Haghnegahdar, ${ }^{1}$ and Shahram Bolandparvaz ${ }^{1}$
}

\author{
${ }^{1}$ Trauma Research Center, Shiraz University of Medical Sciences, Shiraz, IR Iran \\ ${ }^{2}$ Student Research Committee, Shiraz University of Medical Sciences, Shiraz, IR Iran \\ ${ }^{3}$ Department of Surgery, Shiraz Medical School, Shiraz University of Medical Sciences, Shiraz, IR Iran \\ ${ }^{4}$ Department of Epidemiology, Research Center for Health Sciences, School of Health, Shiraz University of Medical Sciences, Shiraz, IR Iran \\ "Corresponding author: Mohammad Ghorbani, Department of Epidemiology, School of health, Razi Street, Shiraz, IR Iran. Tel: +98-7117251001, Fax: +98-7117251009, E-mail: \\ ghorbani_epi@yahoo.com
}

Received 2015 October 12; Revised 2016 January 04; Accepted 2016 February 23.

\begin{abstract}
Background: Epidemiology of cervical spine fractures (CSfx) in trauma patients of general population is not yet exclusively known. Objectives: The purpose of this study was to evaluate the epidemiology of CSfx in trauma patients.

Patients and Methods: Data from trauma patients admitted in the emergency room (ER) of Shiraz Shahid Rajaei hospital during the 3.5 years period from September 22, 2009 to March 21, 2013, were gathered. All trauma patients with CSfx and/or spinal cord injuries were included in the study. The time of the trauma, mechanism of trauma, injury position, and incidence of cervical spine fractures in the patients were recorded.

Results: A total of 469 patients met the inclusion criteria. The mean age of the patients was 34.7 years old, with a minimum age of 16 years old and a maximum age of 89 years old. Young adults were most frequently affected. Out of 469 cases, 368 patients (78.47\%) were male and 101 (21.53\%) were female. We had a total of 17 SCI cases among our patients (3.62\%), out of which 5 (29.41\%) were deceased. The total number of deaths in our study was 29 (6.18\%); $5(17.24 \%)$ with SCI and $24(82.76 \%)$ without SCI.

Conclusions: This study demonstrated that most victims of CSfx in our region are 16 to 40 years of age. A male predominance was observed, and motor vehicle collisions were the most frequent trauma mechanism leading to cervical spine injury (mostly due to car rollover accidents), with falls as the second most frequent. The rate of SCI in our study was $3.62 \%$ of all cases and the mortality rate was $6.18 \%$.
\end{abstract}

Keywords: Cervical Spinal Fractures, Spinal Cord Injury, Trauma, Epidemiology

\section{Background}

Documentation of trauma injuries plays a great role in the prevention and management of trauma by giving us clues and general overviews of trauma injuries. It helps to expand the initiative of trauma injury control and prevention in our area, build up new protocols of trauma management, facilitate our trauma centers, and utilize our resources more efficiently (1-3).

Speaking of the trauma documentation, epidemiology of traumatic cervical spine fractures (CSfx) is not yet well-known among the general population (4). Searching among published papers and literature, we found a few articles, including a 1996 study performed by Hu et al. in Canada that described the incidence of spine fractures in a general population. They estimated this incidence rate to be $64 / 100,000(5,6)$. Based on what they promulgated, the incidence rate of cervical spine fractures can be estimated to be $12 / 100,000$.

Timely spinal immobilization and prompt detection of these injuries during primary and secondary survey is important as this can help to avoid additional injury to the spinal cord. Identification and assessment of cervical spine injury during initial trauma evaluation is challenging as patients often present with a decreased level of consciousness because of concurrent head injury, sedative and analgesic medication, or endotracheal intubation. Clinical decision rules in these circumstances are then helpful tools for proper management (7).

CSfx has more male victims than females (8-17). Agewise, the highest incidence rate is reported to be among patients aged 15 to 45 years, with a second peak in those aged 65 to 80 years $(8,9,12,14,18-20)$.

Most articles report motor vehicle accidents to be the most common mechanism of injury leading to cervical spine injury $(8,13,17,21,22)$. Some others report falls as the most frequent $(15,18,19)$. Spinal cord injury (SCI) is reported to occur in 10 to $50 \%$ of cases of $\operatorname{CSfx}(5,6,8,14,16$, $18,19,22,23)$. 
However, it must be borne in mind that the abovementioned relationships shown between CSfx and gender, age, trauma mechanism, head injury, and SCI are extracted from studies of subpopulations of trauma patients, hence not attributable to the general population.

\section{Objectives}

Hereby, we performed a study retrospectively on data collected from the medical records in the data registry of the Shahid Rajaei hospital, Shiraz, Iran, to determine the incidence of CSfx in a general population in all age ranges and both genders, and to show and describe different mechanisms of trauma leading to SCI. Also, as a secondary goal, we would also be able to compare our data with other centers in our country or even with international data available to provide a more helpful outlook of our situation.

\section{Patients and Methods}

This study was actually a data extraction from classified data of trauma patients admitted in the emergency room (ER) of Shiraz Shahid Rajaei hospital, a level I trauma center in Southwest Iran with an ongoing $24 \mathrm{hr}$ accessible ER which with a huge load of trauma patients monthly, during the 3.5 years period from September 22, 2009 to March 21, 2013.

The primary outcome was CSfx and dislocations, or cervical SCI. All trauma patients with CSfx and/or spinal cord injuries were included in the study. Patients with nontraumatic brain injury, minor blunt and penetrating injuries, and single uncomplicated limb injuries were excluded. Also, injuries to the brachial plexus; trauma to other parts of the vertebrae such as disc, spinous, and transverse processes; nerve root; and strains of the spine were all excluded.

All demographic data was gathered from the data bank. The time of the trauma, mechanism of trauma, injury position, and existence of SCI in patients were also recorded. The Statistical Package for Social Science, SPSS for Windows, version 16.0 (SPSS, Chicago, IL, USA) was used for data analysis.

\section{Results}

Over a period of 3.5 years, 469 patients, who were admitted in the ER of Shahid Rajaei hospital, met the inclusion criteria by having a cervical spine fracture, which was found in the primary and secondary survey. The mean age of patients was 34.68 years old, with a minimum age of 16 years old and a maximum age of 89 years old.

Data of each year of the study was separately analyzed alongside a total study population survey. Of course, since we gathered only six months of data in 2009, the total number of cases was less than in other years. Therefore, we did not aim to compare all four years' statistics together.

As it can be seen in the Table 1, young people comprised the largest portion of the cases. Out of 469 cases, 368 patients (78.47\%) were male and $101(21.53 \%)$ were female.

We had a total of 17 SCI cases among our patients (3.62\%), out of which 5 (29.41\%) were deceased. Furthermore, the total number of deaths was 29 in our study (6.18\%); 5 (17.24\%) with SCI and 24 (82.76\%) without SCI. Separate data about SCI and death cases of each year are shown in Figure 1 and Table 2.

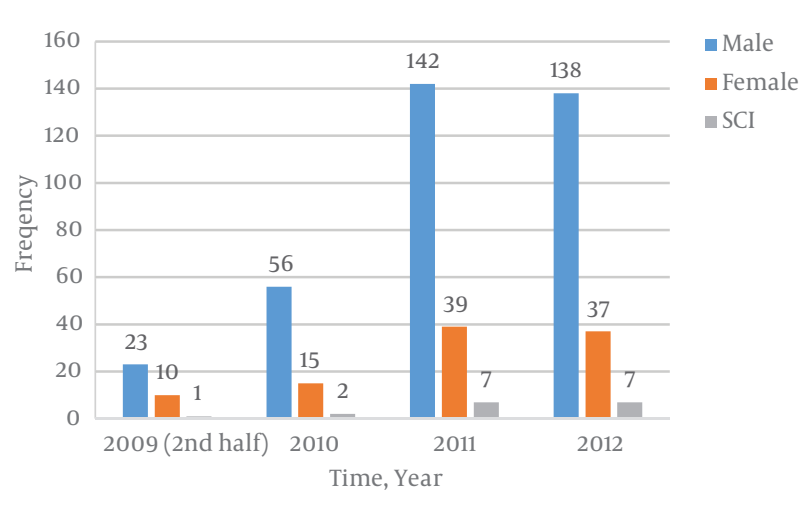

Figure 1. Number of Victims of Each Gender Along With the Total Number of SCI in Each Year of Study

As mentioned earlier, we also took record of the victims' position and situation during motor vehicle accidents so that we would be able to see that most of our victims, as shown in Table 3, were four-wheel vehicle riders (64.9\%). We also had 19 cases (4.05\%) of CSfx due to fall from a determined or unknown height (Table 3).

Other types of trauma leading to CSfx in our study were stab wounds, fights, blunt trauma with objects, and gunshot wounds, among which blunt trauma with solid objects constituted the most cases (50\%) (Table 3). In Table 3, the number of patients does not total 469 because we had missing data in 21 patients.

As one of the main purposes of our study, we were able to classify our cases according to their various mechanisms of trauma. The most common mechanisms of trauma was car rollover (182 cases, $46.78 \%$ ), followed by car-to-car accidents (97 cases, 24.93\%) and-car tomotor accidents (57 cases, $14.65 \%$ ) (Table 4 ). 
Table 1. Number of Cases in Divided Age Ranges in Each Year of Study

\begin{tabular}{|c|c|c|c|c|}
\hline Age Range & 2009 (2nd Half) & 2010 & 2011 & 2012 \\
\hline $15-25$ & 8 & 22 & 56 & 62 \\
\hline $26-35$ & 13 & 20 & 51 & 40 \\
\hline $36-45$ & 2 & 13 & 28 & 22 \\
\hline $46-55$ & 3 & 15 & 25 & 24 \\
\hline $56-65$ & 3 & 5 & 12 & 17 \\
\hline $66-75$ & 2 & 4 & 6 & 7 \\
\hline $76-85$ & 0 & 1 & 3 & 3 \\
\hline $86-95$ & 2 & 0 & 0 & 0 \\
\hline Total & 33 & 80 & 181 & 175 \\
\hline
\end{tabular}

Table 2. Number of Expired Cases in Each Year Along With Number of Spinal Cord Injury (SCI) Among Them

\begin{tabular}{|c|c|c|c|c|c|}
\hline & 2009 (2nd Half) & 2010 & 2011 & 2012 & Total \\
\hline Number of Deaths & 1 & 8 & 12 & 8 & 29 \\
\hline Number of Deaths having SCI & 0 & 1 & 2 & 2 & 5 \\
\hline
\end{tabular}

Abbreviation: SCI, spinal cord injury.

Table 3. Number of CSfx Victims of Fall, Motor Vehicle Accident, and Other Causes in Each Year of Study

\begin{tabular}{|c|c|c|c|c|c|}
\hline & 2009 & 2010 & 2011 & 2012 & Total \\
\hline 4-wheel vehicle rider & 24 & 40 & 92 & 90 & 246 \\
\hline Motorcyclist & 3 & 18 & 35 & 37 & 93 \\
\hline Pedestrian & 2 & 7 & 8 & 23 & 40 \\
\hline Falls from determined height & 3 & 3 & 1 & 0 & 7 \\
\hline Falls from unknown height & 0 & 5 & 22 & 19 & 46 \\
\hline Stab Wound & 0 & 1 & 1 & 0 & 2 \\
\hline Fight & 0 & 2 & 2 & 1 & 5 \\
\hline Blunt trauma with objects & 0 & 2 & 5 & 1 & 8 \\
\hline Gun Shot Wound & 0 & 0 & 0 & 1 & 1 \\
\hline
\end{tabular}

\section{Discussion}

Assessments of the epidemiology of trauma and the recording and classification of this type of data have been done worldwide in recent decades. It will certainly guide us through a wide variety of choices in trauma care and management. In developing countries, it is critical to know and predict how to use limited facilities to be able to benefit all patients. This sort of data classification may also be useful in helping us make comparisons among statistics from different trauma centers, or even international data presented in other studies.

In our study, we observed a male predominance among our patient population (78.5\%). This could mostly be due to the engagement of the male gender in more hazardous vocations; their tendency to do their handwork themselves at home or work, even if they do not have the expertise required; or the fact that males constitute more vehicle riders than females in Iran. Moreover, some other studies in different countries with varying cultures have also shown the same results (8-17).

As one could notice, number of cases has increased in the last years, compared to the first two years. This could be due to either an increase in the number of motor vehicles and cyclists in our city or the progress in our data gathering system, which has covered more cases than the past. 
Table 4. Distribution of Mechanism of Injury in Vehicle Accidents (only) in Each Year of Study

\begin{tabular}{|c|c|c|c|c|}
\hline & 2009 & 2010 & 2011 & 2012 \\
\hline Bicycle to pedestrian & 0 & 0 & 0 & 0 \\
\hline Motorcycle to pedestrian & 0 & 2 & 4 & 1 \\
\hline Car to pedestrian & 1 & 6 & 14 & 22 \\
\hline Heavy vehicle to pedestrian & 0 & 2 & 0 & 0 \\
\hline Bicycle to Motorcycle & 0 & 0 & 0 & 0 \\
\hline Car to Bicycle & 0 & 0 & 0 & 0 \\
\hline Heavy vehicle to Bicycle & 0 & 0 & 0 & 0 \\
\hline Motor to Motor & 1 & 2 & 5 & 0 \\
\hline Car to Motor & 2 & 8 & 24 & 23 \\
\hline Motor Turnover & 0 & 7 & 6 & 14 \\
\hline Car to Car & 8 & 16 & 37 & 36 \\
\hline Car Turnover & 15 & 22 & 55 & 90 \\
\hline Car to Objects & 0 & 0 & 0 & 0 \\
\hline Bus Turnover & 1 & 0 & 0 & 0 \\
\hline Total & 29 & 65 & 145 & 150 \\
\hline
\end{tabular}

The latter could be counted as one of our limitations, since our data gathering protocols have not been perfect in the past.

The age distribution of our cases reveals the highest frequency of cervical spine fracture among those aged 16 - 40 years. We also found a decreasing incidence with increasing age. Other former reports found the third decade of life, or ages of 15 - 45 years, to be more strongly associated with an increased risk of cervical spine injury as well $(8,9$, $14,18,20)$. Some authors, however, have also reported a second peak at age $65-80(12,19,20)$, which was not that high among our cases. Some recent reports indicate a trend during recent decades of an increasing median age of spine trauma patients and a shift towards falls as the most frequent mechanism of injury $(11,17)$.

Our study showed that, in our region, motor vehicle collisions are the most frequent trauma mechanism leading to cervical spine injury, with falls the second most frequent $(8,13,17,21,22)$. A number of authors report the same findings that we do regarding trauma mechanism $(15,18$, 19). Among motor vehicle accidents, the most common mechanisms of trauma was car rollover (182 cases, $46.78 \%$ ), followed by car-to-car accidents (97 cases, 24.93\%) and carto-motor accidents ( 57 cases, $14.65 \%$ ). However, a study performed in Norway showed the contrary, as fall-related injuries were observed in more than half of their patients (24).
The incidence of cervical SCI in the setting of a cervical spine fracture is reported to be $12 \%-50 \%(5,6,8,14,16,18,19$, $22,23)$. In Hu's study, this incident accounted for $10 \%$ of the patient population. But, surprisingly, the incidence rate of SCI in our cases was just 3.62\%.

\subsection{Conclusions}

Our retrospective study showed numbers of CSfx cases in the period of 3.5 years. This data demonstrated that most victims in our region are 16 to 40 years old and the incidence decreases with rising age. A male predominance was observed, and motor vehicle collisions were the most frequent trauma mechanism leading to cervical spine injury, with falls the second most frequent. The rate of SCI in our study was $3.62 \%$ of all cases and the mortality rate was $6.18 \%$. We suggest other prospective studies for assessing and predicting the risk factors for the CSfx.

\section{Acknowledgments}

Here, we would like to thank Leyla Shayan, M.S, for helping us analyze our data. We would also like to thank the nurses of the emergency room and the staff of the archives department of Shahid Rajaei Hospital, Shiraz, Iran, who cooperated eagerly with us to perform this study. Hereby, we declare that none of the authors of this paper has any financial or other relationships that may lead to conflict of interest. 


\section{References}

1. Sharma BR. The injury scale-a valuable tool for forensic documentation of trauma. J Clin Forensic Med. 2005;12(1):21-8. doi: 10.1016/j.jcfm.2004.08.002. [PubMed: 15763686].

2. Abbasi HR, Mousavi SM, Akerdi AT, Niakan MH, Bolandparvaz S, Paydar S. Pattern of traumatic injuries and Injury Severity Score in a major trauma center in Shiraz, Southern Iran. Bull Emerg Trauma. 2013;1(2 APR):81-5.

3. Mehrpour SR, Nabian MH, Oryadi Zanjani L, Foroughmand-Araabi $\mathrm{MH}$, Shahryar Kamrani R. Descriptive epidemiology of traumatic injuries in 18890 adults: a 5-year-study in a tertiary trauma center in iran. Asian J Sports Med. 2015;6(1):eee23129. doi: 10.5812/asjsm.23129. [PubMed: 25883772].

4. Clayton JL, Harris MB, Weintraub SL, Marr AB, Timmer J, Stuke LE, et al. Risk factors for cervical spine injury. Injury. 2012;43(4):431-5. doi: 10.1016/j.injury.2011.06.022. [PubMed: 21726860].

5. Hu R, Mustard CA, Burns C. Epidemiology of incident spinal fracture in a complete population. Spine (Phila Pa 1976). 1996;21(4):492-9. [PubMed: 8658254].

6. DesPlaines I. Committee on Injury Scaling, Association for the advancement of automotive medicine. The abbreviated injury scale; 1990.

7. Hasler RM, Exadaktylos AK, Bouamra O, Benneker LM, Clancy M, Sieber $\mathrm{R}$, et al. Epidemiology and predictors of cervical spine injury in adult major trauma patients: a multicenter cohort study. J Trauma Acute Care Surg. 2012;72(4):975-81. doi: 10.1097/TA.0b013e31823f5e8e. [PubMed: 22491614].

8. Bayless P, Ray VG. Incidence of cervical spine injuries in association with blunt head trauma. Am J Emerg Med. 1989;7(2):139-42. [PubMed: 2920074].

9. Dryden DM, Saunders LD, Rowe BH, May LA, Yiannakoulias N, Svenson LW, et al. The epidemiology of traumatic spinal cord injury in Alberta, Canada. Can J Neurol Sci. 2003;30(2):113-21. [PubMed: 12774950].

10. Hasler RM, Exadaktylos AK, Bouamra O, Benneker LM, Clancy M, Sieber $\mathrm{R}$, et al. Epidemiology and predictors of spinal injury in adult major trauma patients: European cohort study. Eur Spine J. 2011;20(12):217480. doi: 10.1007/s00586-011-1866-7. [PubMed: 21644051].

11. Holly LT, Kelly DF, Counelis GJ, Blinman T, McArthur DL, Cryer HG. Cervical spine trauma associated with moderate and severe head injury: incidence, risk factors, and injury characteristics. J Neurosurg. 2002;96(3 Suppl):285-91. [PubMed: 11990836].
12. Jackson AB, Dijkers M, Devivo MJ, Poczatek RB. A demographic profile of new traumatic spinal cord injuries: change and stability over 30 years. Arch Phys Med Rehabil. 2004;85(11):1740-8. [PubMed:15520968].

13. Robertson A, Giannoudis PV, Branfoot T, Barlow I, Matthews SJ, Smith RM. Spinal injuries in motorcycle crashes: patterns and outcomes. $J$ Trauma. 2002;53(1):5-8. [PubMed: 12131381].

14. Sterne JA, White IR, Carlin JB, Spratt M, Royston P, Kenward MG, et al. Multiple imputation for missing data in epidemiological and clinical research: potential and pitfalls. BMJ. 2009;338:b2393. doi: 10.1136/bmj.b2393. [PubMed: 19564179].

15. Canadian C, C. Spine Study Group . Canadian C-Spine Rule study for alert and stable trauma patients: I. Background and rationale. CJEM. 2002;4(2):84-90. [PubMed: 17612425].

16. Thompson WL, Stiell IG, Clement CM, Brison RJ, Canadian CRSG. Association of injury mechanism with the risk of cervical spine fractures. CJEM. 2009;11(1):14-22. [PubMed: 19166635].

17. Tian HL, Guo Y, Hu J, Rong BY, Wang G, Gao WW, et al. Clinical characterization of comatose patients with cervical spine injury and traumatic brain injury. J Trauma. 2009;67(6):1305-10. doi: 10.1097/TA.0b013e31819db57c. [PubMed: 20009682].

18. Soicher E, Demetriades D. Cervical spine injuries in patients with head injuries. BrJ Surg. 1991;78(8):1013-4. [PubMed: 1913094]

19. Mulligan RP, Friedman JA, Mahabir RC. A nationwide review of the associations among cervical spine injuries, head injuries, and facial fractures. I Trauma. 2010;68(3):587-92. doi: 10.1097/TA.ob013e3181b16bc5. [PubMed: 19996802].

20. Smith JA, Siegel JH, Siddiqi SQ. Spine and spinal cord injury in motor vehicle crashes: a function of change in velocity and energy dissipation on impact with respect to the direction of crash. J Trauma. 2005;59(1):117-31. [PubMed: 16096551]

21. Krug EG, Sharma GK, Lozano R. The global burden of injuries. Am JPublic Health. 2000;90(4):523-6. [PubMed:10754963]

22. Heidari P, Zarei MR, Rasouli MR, Vaccaro AR, Rahimi-Movaghar V. Spinal fractures resulting from traumatic injuries. Chin J Traumatol. 2010;13(1):3-9. [PubMed: 20109360].

23. Varma A, Hill EG, Nicholas J, Selassie A. Predictors of early mortality after traumatic spinal cord injury: a population-based study. Spine (Phila Pa 1976). 2010;35(7):778-83. doi: 10.1097/BRS.0b013e3181ba1359. [PubMed: 20228715].

24. Fredo HL, Rizvi SA, Lied B, Ronning P, Helseth E. The epidemiology of traumatic cervical spine fractures: a prospective population study from Norway. Scand J Trauma Resusc Emerg Med. 2012;20:85. doi: 10.1186/1757-7241-20-85. [PubMed: 23259662]. 